Rev. Psicol. (Arequipa. Univ. Catól. San Pablo) / Año 2021 / Vol 11 / № 1 / pp. 145-167

ISSN 2306-0565 versión impresa / ISSN 2311-7397 versión on line

\title{
Una aproximación a la clasificación de heridas afectivas
}

An Approach to the Clasification of Affective Wounds

Paola Rodríguez Pacas

Centro de Estudios Areté, Medellín, Colombia

iD https://orcid.org/oooo-0003-3215-2650

Humberto Del Castillo Drago

Centro de Estudios Areté, Medellín, Colombia

(iD https://orcid.org/oooo-0oo2-9135-8862

Correspondencia: psicologohumberto@centroarete.org

Mónica Caballero Andrade

Centro de Estudios Areté, Medellín, Colombia

(iD https://orcid.org/oooo-0oo2-2732-2566

\section{Resumen}

Las heridas afectivas son situaciones o acontecimientos fuertes que producen una alteración en la afectividad de la persona. Desde el modelo de la Psicoterapia de la Reconciliación (Del Castillo, 2016), se ha visto que dichas heridas pueden aparecer a lo largo de la historia personal, tanto en el ámbito familiar como en otras áreas vitales. Este artículo procura hacer una revisión de las manifestaciones psicológicas que las heridas afectivas tienen en la persona adulta, y además, presentar una clasificación y definición de las mismas. En general, los resultados indican que las heridas afectivas sí tienen consecuencias clínicamente significativas en el componente psicológico, y que estas pueden ser clasificadas para facilitar su identificación durante el proceso psicoterapéutico.

Palabras clave: Psicoterapia, trauma, herida afectiva, reconciliación personal.

\begin{abstract}
Affective wounds are powerful situations or events that produce an alteration in the affectivity of the person. From a Reconciliation Psychotherapy model (Del Castillo, 2016), it has been seen that these wounds can appear throughout one's personal history, both in the family environment and in other vital areas. This article attempts to outline the psychological manifestations that affective
\end{abstract}


injuries have in adults, and also present a classification and definition of these emotional wounds. Furthermore, it seeks to define and classify them according to the conditions in which they appear. In general, the results indicate that affective wounds do have clinically significant consequences on the psychological functioning, and that these consequences can be classified in order to facilitate their identification during the therapeutic process.

Keywords: Psychotherapy, trauma, affective wound, personal reconciliation.

\section{Introducción}

\section{Noción de trauma psicológico y heridas afectivas}

En la actualidad, se ha visto cada vez más un cambio en la actitud de la sociedad frente a la salud mental, particularmente frente a temas en los que su "felicidad" o bienestar psicológico se ve comprometido. En su libro, Norman Bradburn (1969) argumenta que, cuando se habla de bienestar psicológico, no se pretende indagar por la presencia o ausencia de diagnósticos psiquiátricos, sino describir los problemas cotidianos a los que se enfrentan las personas mientras luchan por cumplir sus metas de vida. Este autor distingue el afecto positivo del afecto negativo, e investiga cómo los macro o micro-cambios sociales e interpersonales influyen en el bienestar subjetivo de las personas. Más adelante, Carol Ryff (1989), basada en la teoría de Bradburn (1969), busca establecer los índices del funcionamiento personal positivo mediante la operacionalización de las seis dimensiones correspondientes al bienestar psicológico: aceptación personal, relaciones interpersonales positivas, autonomía, dominio ambiental, propósito de vida y crecimiento personal. A día de hoy, en la práctica clínica se ve cada vez más que las personas tienen serias dificultades para vivir adecuadamente muchas de las dimensiones del bienestar psicológico propuestas por Ryff (1989). Muchas veces, llegan a consulta sin saber a qué se atribuye su malestar.

Generalmente, en la Psicología se suele recurrir a la palabra "trauma" para describir esos acontecimientos que generan sufrimiento en la persona, y que en el CIE-11 (2018) se definen como: «cualquier exposición a una situación estresante de naturaleza excepcionalmente amenazante u horrorizante que probablemente producirá un malestar profundo en la mayoría de las personas» (p. 11). Sin embargo, el trauma y sus consecuencias dependen de tantos factores, que se ha tenido que ampliar el repertorio de situaciones potencialmente traumáticas, especialmente porque se ha visto que las manifestaciones traumáticas no se dan uniformemente en toda la población (Pereda et al., 2013). En otras palabras, el concepto de "trauma" o de "experiencia traumática” varía según el umbral de vulnerabilidad, los recursos de afrontamiento y el entorno socio-cultural de cada persona.

La noción de trauma sevuelve relevanteen la actualidad debido a que es una situación que puede generar un cambio significativo 
en la afectividad de la persona, siendo definido este último concepto por Sarráis (2016) como:

La facultad psíquica por la que las vivencias conscientes nos afectan. Permite convertir en experiencia interna (vivencia) cualquier contenido de conciencia y, así, experimentar íntimamente las realidades exteriores y las de uno mismo. Su función es dotar de significado personal la propia experiencia. (p. 141)

Samper (2016) afirma que el trauma psicológico afecta a todo el ser de la persona, por lo cual sus manifestaciones y consecuencias se podrán observar a nivel psíquico y corporal. Figueroa, Cortés, Accatino y Sorensen (2016) añaden que si los traumas más graves no se manejan, es posible que dejen diversas secuelas psicológicas, como Trastorno de Estrés Postraumático (TEPT), trastornos de ansiedad, trastornos del humor, entre otros. Adicionalmente, Figueroa et al. (2016) argumentan que las secuelas emocionales de los traumas generan una variedad de problemáticas psicosociales que pueden dificultar la realización y el bienestar de la persona, como serían los problemas maritales, la disfunción familiar, la baja laboral, el abuso de sustancias y el suicidio.

Sin embargo, es llamativo ver cómo en la actualidad, existen situaciones que, si bien no catalizan la aparición inminente de un trastorno psiquiátrico tras su vivencia, alteran la afectividad de la persona y llegan a considerarse "eventos potencialmente traumáticos”. Según Shapiro (2013), este tipo de eventos tienen la capacidad de generar pensamientos intrusivos, sentimientos de culpa, sueños perturbadores y aislamiento social. Además, argumenta que estas alteraciones pueden aparecer aunque no haya un diagnóstico de Trastorno de Estrés Postraumático, y que son una señal de que la persona tiene recuerdos no procesados que están generando el malestar. Los eventos potencialmente traumáticos pueden aparecer en distintas etapas del desarrollo, y debido al sufrimiento que traen consigo, pueden generar creencias y actitudes disfuncionales que producen un daño en la dimensión biológica, psicológica y espiritual de la persona, y sientan las bases para el desarrollo de patologías psiquiátricas graves. Shapiro (2013) resalta que, hasta los eventos "aparentemente menores" desde una perspectiva de adultos, pueden ser las causas del malestar que vive la persona en la actualidad, y Salgado (2014) apoya esta idea, argumentando que: «existe un gran vacío existencial y carencias afectivas que se traducen en una mayor prevalencia a nivel mundial de cuadros como la ansiedad, la depresión y estrés que lamentablemente forman parte de la vida, desde edades muy tempranas» (p. 123).

Desde el modelo del Centro Areté, estos eventos se denominan "heridas afectivas", y corresponden al principal elemento de intervención de la Psicoterapia de la Reconciliación (Del Castillo, 2016). Se entiende que una herida afectiva es una situación o acontecimiento fuerte que altera y afecta todo el componente afectivo de la persona, produciendo un daño en la dimensión física, psicológica y espiritual de la misma (Del Castillo, 
2016). Estas pueden darse por la no satisfacción de la necesidad afectiva, o por una satisfacción exagerada de la misma. En concreto, Del Castillo (2016) afirma que una inadecuada satisfacción de la necesidad afectiva produce heridas que, si no se cuenta con los recursos psicológicos o con una capacidad de resiliencia para afrontarlas, pueden generar graves afecciones y trastornos en la persona. No obstante, sin importar que se den por falta o por exceso, cuando las heridas afectivas aparecen desde la infancia, pueden catalizar la formación de esquemas cognitivos maladaptativos, creencias nucleares disfuncionales y, eventualmente, trastornos mentales. Shapiro (2013) expone que estos esquemas, creencias y trastornos mentales son únicamente el síntoma, no la causa, de la problemática del consultante. En definitiva, la causa serían los recuerdos o eventos que no han sido procesados adecuadamente, y se convierten en los agentes que generan reacciones desproporcionadas, y conductas destructivas con uno mismo y con los demás.

\section{La relevancia clínica de las heridas afectivas}

Se ha visto que cuando las heridas están ligadas a acontecimientos en la historia personal, estas producen un marcado dolor y sufrimiento (Del Castillo, 2016) que, dependiendo de la vulnerabilidad de cada uno, pueden generar pensamientos, emociones y conductas disfuncionales y perjudiciales en las personas. Sarráis (2016) añade a esta idea que, si bien la afectividad es fuente de conocimiento que nos ayuda a saber si la realidad nos afecta positiva o negativamente, tiene una doble función: sirve como un motor de la conducta, impulsa a tener comportamientos y acatar acciones orientadas a mantener o aumentar los afectos positivos, y a suprimir o reducir los negativos. En otras palabras, los afectos muchas veces entran en conflicto con la fuerza de la voluntad, y pueden llevar a la persona a desarrollar y mantener conductas que le perjudican en un intento de reducir su malestar.

De acuerdo a Bourbeau (2014), hay algunos síntomas que delatan la presencia de esta dinámica, como son los síntomas ansiosos, miedos, sentimientos de culpa, vergüenza e inseguridad, reacciones desproporcionadas ante los eventos, poca valoración personal, las compulsiones y, en general, un patrón negativo de conducta. Por tanto, se vuelve relevante intervenir en estos choques o heridas en la afectividad para que la persona no se vea envuelta en patrones vitales disfuncionales.

Si bien Bourbeau (2014) ha logrado describir adecuadamente algunas de las manifestaciones de las heridas afectivas, se encuentra que aún no hay investigaciones o revisiones teóricas que describen las consecuencias psicológicas que corresponden a la presencia de cada tipo de herida. Por otro lado, aunque Bourbeau (2014) ha clasificado las heridas en cinco tipos (el rechazo, el abandono, la humillación, la traición y la injusticia), en la revisión literaria no se ha concertado una 
clasificación definitiva, observándose cierta discrepancia entre los autores. Por lo que, es necesario realizar una clasificación que abarque todos los tipos de heridas, así como el impacto de cada una de ellas en las personas que las vivencian.

\section{Objetivos}

El objetivo principal de esteartículo de revisión es mostrar el estado actual de conocimiento acerca de las heridas afectivas propuestas por el modelo de la Psicoterapia de la Reconciliación, así como describir las consecuencias psicológicas que tienen en la persona adulta. Además, como objetivos específicos del artículo se enumeran los siguientes: 1) presentar la actual clasificación de heridas afectivas y sus definiciones, e 2) identificary sintetizar los principales impactos que tienen las heridas afectivas en el componente psicológico del adulto.

\section{Método}

\section{Protocolo y registro}

El presente trabajo tendrá como objetivo proponer una clasificación de las heridas afectivas, así como sus respectivas consecuencias en la dimensión psicológica de la persona. Con este objetivo en mente, se decidió elaborar un artículo de revisión basado en el formato IMRyD, propuesto por Instituto Americano de Estándares Nacionales en 1972 (Camps, 2007).

En concreto, el formato IMRyD es el acrónimo de los apartados que conforman la estructura general del artículo científico: introducción, métodos, resultadosy discusión (García y Castellanos-Verdugo, 2007). Según García y Castellanos-Verdugo (2007), esta estructura está diseñada para cualquier estudio que busca describir paso a paso el proceso de investigación llevado a cabo, $y$ «ayuda a guiar a directores, revisores y lectores en la consulta, comprensión y evaluación de un informe de una investigación científica» (García \& Castellanos-Verdugo, 2007, p. 3).

\section{Fuentes de información}

Esta revisión se basó en los artículos y estudios recopilados a partir de las siguientes fuentes:

UNIKA. Base de datos unificada desarrollada por la Universidad de Navarra. Esta herramienta hace una síntesis de los catálogos de la Biblioteca de la Universidad de Navarra, haciendo uso de los portales de DADUN y de SABIO (Universidad de Navarra, 2011). La búsqueda en esta fuente se comenzó el 11 de junio de 2020 y finalizó el 16 de septiembre de 2020.

Science Direct. Base de datos que da acceso por suscripción a publicaciones científicas y académicas en el ámbito de la medicina y la salud. La biblioteca electrónica contiene monografías académicas, guías técnicas y otros textos de referencia en texto completo. La búsqueda en esta fuente se comenzó el 11 de junio de 2020 y finalizó el 16 de septiembre de 2020 .

SciELO. Este sitio es la aplicación modelo de la metodología SciELO, con la cual se pretende divulgar por vía electrónica publicaciones periódicas y científicas 
que son compatibles con las iniciativas internacionales más importantes. Además, también pretende ser una base de datos bibliográfica para literatura científica y producir indicadores para subsidiar estudios de bibliometría, informetría y cienciometría (Packer et al., 2001).

EBSCOhost: es una base de datos que ofrece textos completos, referencias y resúmenes de publicaciones periódicas, científicas y académicas, de diferentes áreas de las ciencias y humanidades (Plasencia, 2008). La búsqueda en esta fuente se comenzó el 11 de junio de 2020 y finalizó el 16 de septiembre de 2020 .

\section{Búsqueda}

En la búsqueda del presente trabajo, se procuró incluir publicaciones tanto en español como en inglés, publicadas desde el 2010 hasta la actualidad, y cuyos contenidos estuvieran directamente relacionados al concepto de "herida afectiva" que intentábamos desarrollar. Las palabras clave que se utilizaron en los distintos buscadores son las siguientes: "Efectos psicológicos abandono físico", "Efectos psicológicos abandono psicológico", "Consecuencias psicológicas maltrato físico", "Consecuencias psicológicas maltrato psíquico", "Consecuencias del amor posesivo", "Conflictos conyugales efectos", "Consecuencias violencia intrafamiliar", "Consecuencias muerte de familiar", "Consecuencias psicológicas abuso sexual", "Efectos experiencias sexuales tempranas" , "Early sexual experiences",
"Consecuencias fracaso en amistades", "Efectos del duelo", "Consecuencias psicológicas ruptura amorosa”, "Consecuencias del acoso", "Bullying", "Bullying consequences", "Consecuencias psicológicas fracaso académico", "Consecuencias psicológicas fracaso laboral", "Fracaso académico y motivación", "Fracaso académico y autoestima", "Limitaciones intelectuales y motivación”, "Autoconcepto negativo", "Sentimientos de inutilidad psicología", "Consecuencias rechazo al cuerpo", “Consecuencias estigmatización”, "Consecuencias psicológicas aborto"

Cada término arrojó distintas cantidades de resultados, y se fueron eliminando automáticamente los artículos repetidos.

\section{Criterios de inclusión y exclusión}

Para este artículo, se seleccionaron artículos que cumplieran con los siguientes criterios de inclusión:

a) Artículos o investigaciones que fueron publicadas hace diez años o menos.

b) Publicaciones que estudian las consecuencias psicológicas de las heridas afectivas con personas mayores de 18 años.

c) Estudios que plantean una definición sobre los conceptos que se buscaban desarrollar en profundidad.

d) Investigaciones sobre las consecuencias que las heridas afectivas propuestas pueden generar en el bienestar psicológico de las personas. 
Con respecto a los criterios de exclusión, se tomaron en cuenta los siguientes:

a) Publicaciones de hace más de diez años.

b) Trabajos fin de grado (TFG), trabajos fin de máster (TFM) o tesis doctorales.

c) Estudios que no investigaban los efectos psicológicos de las heridas propuestas.

d) Investigaciones llevadas a cabo en poblaciones específicas (por países, por sexo, por nivel socioeconómico, etc.).

\section{Proceso de extracción de datos y lista de datos}

La extracción de datos de las publicaciones seleccionadas para este artículo se llevó a cabo por medio del análisis a texto completo de cada publicación, con el objetivo de decidir si los estudios cumplían con los criterios de inclusión mencionados en el apartado anterior, o no. Luego de esta revisión, se incorporó la nueva información a las definiciones de heridas afectivas propuestas en el libro, "Reconciliación de la historia personal" (Del Castillo, 2016).

\section{Resultados}

Al día de hoy, las heridas afectivas son clasificadas en dos grandes contextos: heridas familiares y heridas de otros ámbitos. Las heridas afectivas familiares son aquellas que se generan en el núcleo familiar de la persona, por lo que suelen estar relacionadas con figuras importantes de este sistema. La importancia de las heridas familiares radica en que impiden el desarrollo correcto del concepto de sí mismo y de la recta valoración personal. Esto se hace evidente desde la noción de que las heridas afectivas surgen por defecto o por exceso de la satisfacción de una necesidad afectiva (Del Castillo, 2016). En la misma línea, Shapiro (2013) menciona que, aunque no existan recuerdos tangibles de las heridas, puede pasar que hasta los padres que más buscan apoyar y hacer lo mejor para sus hijos, terminan enredándolos en una "red" de síntomas y dolor que los niños no procesan a nivel consciente. Kleponis (2018) añade que muchas son las personas que experimentan rechazo y abandono por parte de sus padres. Otros experimentan abuso, ya sea psicológico, emocional o sexual, que se constituyen en heridas de la familia de origen y que pueden incluir abusos, adicción, muerte, divorcio, abandono y rechazo. El mencionado autor destaca que todos los niños necesitan sentirse amados, seguros, valorados y protegidos por los adultos; al no recibir nada de esto, dichas experiencias pueden ser traumáticas para los niños (Kleponis, 2018).

Ya sea por un exceso o defecto en la satisfacción de las necesidades emocionales, el trato de los padres y la familia con el niño se vuelve un factor influyente en la calidad y el tipo de relaciones que este buscará y tendrá en la adultez. Polaino (2010) afirma que la ausencia del padre, su no 
comparecencia y desencuentro con el hijo, su deslocalización en el hogar, condiciona poderosamente el desarrollo afectivo tan anómalo en los hijos. Un desarrollo nefasto, que hace daño al hijo, genera distancia y rencores con el padre y, lo que es peor, lo incapacita a la larga para hacer feliz a la mujer de su vida. (p. 27)

Se considera importante entender cómo la insatisfacción de las necesidades primordiales de un niño puede generar problemas psicológicos, esquemas maladaptativos, déficits en la personalidad o trampas vitales (Young, 2013). Sin embargo, estas heridas, que se transforman en "patrones" de relación y que configuran la concepción que se tiene de uno mismo y del mundo, eventualmente terminan generandoalgún malestar importante en el sujeto, por lo que se hace relevante identificarlas para, posteriormente, poder aceptarlas, reconciliarlas y vivir una vida virtuosa y plena.

La actual clasificación de las heridas afectivas familiares se presenta a continuación:

Abandono físico: hace referencia a la falta de respuesta de los padres o cuidadores a las necesidades físicas de los hijos, como son el alimento, abrigo, higiene, atención de la salud y cuidado. De acuerdoa Young, Klosko y Janet (2012), el abandono físico también puede hacer referencia a situaciones diversas, como cuando el niño se ve separado de uno de sus cuidadores por un tiempo prolongado, cuando el niño fue criado por niñeras, instituciones o internados; incluyendo, cualquier situación en la que la relación con alguno (o ambos) padres se perdió, y generó, como consecuencia, respuestas de ansiedad, depresión o ira (Young et al., 2012). Suele manifestarse especialmente como una dificultad en las relaciones íntimas, en las que aparecen miedos relacionados a un posible abandono, que gatillan respuestas afectivas y conductuales disfuncionales.

Abandono psicológico: situación en la que los padres juegan con las necesidades emocionales básicas de los hijos, o llevan a cabo comportamientos que son insensibles al nivel de desarrollo de los niños (Zeanah \& Humphreys, 2018). Estos últimos se refieren a cualquier tipo de acto que comprometa la seguridad psíquica, la aceptación, la autoestima o la autonomía del niño; por ejemplo, falta de contacto o cuidado afectivo, causado por la presencia de sentimientos de indiferencia o rencor hacia los hijos. Según Godbout et al. (2019), cuando se presenta esta herida, el niño crece creyendo que es intrínsecamente inaceptable y no merecedor de ser amado, por lo que desarrolla un esquema de vulnerabilidad e impotencia, y termina desarrollando alguna de las siguientes tendencias: ansiedad por abandono o evitación de la intimidad. Por un lado, la ansiedad por abandono representa el miedo que tiene la persona de ser rechazado por los demás, lo cual lleva a que esta se encuentre híper-vigilante a cualquier señal de amenaza en sus relaciones. Por otro lado, la evitación de la intimidad, implica la supresión emocional y la incomodidad con la cercanía, que sirve para reducir la probabilidad de afectos negativos que pueden surgir por rechazo o abandono. Así mismo, esta herida se traduce como un "sentimiento de vacío", sensación que lleva al niño a pensar que 
está destinado a estar solo (Young et al., 2012); manifestándose una incorrecta valoración de sí mismo, e impidiendo la adecuada realización personal (Del Castillo, 2016).

Maltrato físico y psíquico: describe la agresión por parte de uno, o ambos cuidadores, hacia la integridad física o psíquica del resto de los miembros de la familia; en la que tanto los hijos que son maltratados, como sus agresores, sufren como consecuencia de la agresión. Esta tendencia es característica de los padres que tienen un estilo educativo autoritario, en el que la fuerza, las amenazas, los insultos y la intimidación suelen ser usados para educar. Estos aspectos pueden causar graves lesiones y daños psicológicos en los agredidos, a veces propiciando el desarrollo de un trastorno de la personalidad (Del Castillo, 2016). Esta herida afectiva puede repercutir en un inadecuado sentido de valoración personal como resultado de la privación de singularidad que vivió la persona, y manifiesta sus consecuencias tanto en la vida adulta de la víctima como en la sociedad en la que esta se desenvuelve ya que afecta las relaciones interpersonales (Forero et al., 2010).

\section{Super-corrección e hiperexigencia:} tendencia de padres o cuidadores que hacen vivir a sus hijos bajo el peso del perfeccionismo; que a diferencia de la búsqueda de la perfección, que es un "valor" humano, el perfeccionismo es un hábito riguroso, exteriorista, y se queda en la perfección por la perfección; es por eso que este tipo de herida se vuelve un impedimento para el desarrollo personal y para la recta valoración de sí mismo (Del Castillo, 2016). La persona con una herida afectiva basada en la super-corrección tiene dificultades para adaptarse, es inflexible y rígida, se le hace imposible vivir con espontaneidad y es incapaz de improvisar soluciones, vive constantemente bajo el peso de la culpa, ya que se esfuerza a diario para alcanzar la perfección, que en realidad, es imposible.

\section{Ausencia de confianza y comunica-} ción: hace referencia a la falta de comunicación entre padres e hijos, que impide el desarrollo de la confianza y la comunicación entre los miembros de la familia. Esta tendencia suele aparecer en los casos de padres autoritarios, y dificulta el desarrollo del compañerismo, el afecto, la confianza y unión en la familia. De esta forma, el niño crece en un ambiente en el que no han sido escuchados y en el que no se han sentido importantes, por lo que la comunicación familiar continúa siendo fluctuantey fría en la adolescencia y en la adultez.

\section{Ausencia de reconocimiento y afecto:} ambiente familiar en el que los logros, cualidades y características de sus miembros no son valorados y no reciben el reconocimiento que se merecen. Esta herida hace que los hijos crean que no serán reconocidos ni amados, hagan lo que hagan; en este sentido, el aprendizaje y los logros se ven subordinados al afecto de los padres, y eliminan la seguridad que los hijos deberían desarrollar a partir del cariño incondicional de sus padres. 
Crítica negativa: herida que surge cuando los comentarios o críticas de los padres hacia sus hijos suelen ir acompañadas de intensas respuestas emocionales negativas (Del Castillo, 2016), que casi siempre generan una inadecuada valoración personal, sentimientos de inferioridad y baja motivación en su vida diaria. La crítica negativa también enseña a los niños que los errores son imperdonables y rígidos, ya que no propone ninguna solución que fomente el bien del hijo y de la familia. Al ser criticado y comparado con los demás, esta herida dificulta al hijo a superar sus fracasos, ya que interpreta las críticas de sus padres como una falta de confianza en él y en sus habilidades.

Amor posesivo: esta herida surge cuando el niño rompe con la fase de simbiosis, al darse cuenta que no necesita del adulto para relacionarse con la realidad, ya que materializa la idea de que es un alguien (Papalia y Martorell, 2012), pero la madre no acepta conscientemente esta "ruptura", generando una relación de dependencia mutua y el amor hacia el hijo se podría convertir en un amor posesivo. Young et al. (2012) postula que son madres que refuerzan las conductas dependientes y desalientan las independientes, privando a los hijos de la libertad o el apoyo para ser autónomos. La manifestación de esta herida se da en situaciones en las que la persona debe tomar el control de su vida, pero se ve incapaz de hacerlo ya que no se encuentran las habilidades que se necesitan para afrontarlas por sí solo, y recurre a la aprobación o consejo de los demás, originando sentimientos de incapacidad en la persona.

Conflictos conyugales: Los conflictos conyugales se puede adoptar en distintas formas y afectar a los niños dependiendo de cómo los padres decidan resolver el conflicto; estos pueden ser constructivos o destructivos, siendo los primeros aquellos que lo resuelven con amor, comprensión y compromiso; y los últimos aquellos que lo resuelven con hostilidad y agresión, los cuales suelen generar emociones negativas en los hijos, ya que perciben que la seguridad de su familia está perjudicada (Koss et al., 2011). De esta manera, cuando la estructura afectiva que sostiene el mundo interno del niño se derrumba o se ve amenazada, este puede sufrir alteraciones en su sistema conductual, como intranquilidad, agresión e impulsividad, y en su sistema psicosomático, experimentando síntomas como cefaleas y problemas digestivos; adicionalmente, el sistema emocional se ve afectado de forma notable, predominando los síntomas depresivos y ansiosos (Nuñez et al., 2017). Además, los conflictos conyugales pueden tener un efecto en la idea y el modelo que los niños elaboran de la vida en pareja, poniendo en juego las futuras relaciones personales.

Violencia intrafamiliar: herida que surge cuando la persona creció en un ambiente en el cual hubo algún tipo de acto de poder recurrente dirigido a dominar, someter o agredir física o psicológicamente a uno o más miembros de la familia. Según la investigación de Baader (2014), existe un consenso mundial de que 
la exposición de los niños a cualquier tipo de violencia intrafamiliar trae consigo consecuencias psicológicas negativas. En concreto, parece ser que les genera dificultades para regular el afecto, para la identificación y expresión emocional, y para desarrollar conductas empáticas. Además, perjudica la autoestima de los niños y genera pérdida de autoconfianza, ocasionando a su vez, que los niños desarrollen un miedo crónico que les dificulta adaptarse a diferentes situaciones y ambientes (Baader, 2014). Esta herida también puede llegar a manifestarse mediante conductas agresivas, oposicionistas y antisociales, las cuales perjudican el correcto desarrollo de las relaciones interpersonales. Esta herida afectiva puede repercutir en un inadecuado sentido de valoración personal como resultado de la privación de singularidad que vivió la persona, y manifiesta sus consecuencias tanto en la vida adulta de la víctima como en la sociedad en la que esta se desenvuelve ya que afecta las relaciones interpersonales (Forero et al., 2010).

Del Castillo (2016) afirma que, además de las heridas familiares, se vuelve importante también estudiar y trabajar otros ámbitos de la vida del ser humano donde se pueden generar heridas afectivas. Las heridas de otros ámbitos, entre los que destacan el social, laboral, personal, cultural o económico; también pueden tener una resonancia considerable en el componente afectivo y psicológico de la persona, y son especialmente importantes porque llegan a impedir la realización personal del ser humano. En concreto, se ha visto que si estos sufrimientos no son aliviados, las personas tienen dificultades para vivir alegres y sanas a largo plazo, debido a que el malestar suele volver a aparecer, muchas veces de forma más intensa y extendida (Luciano \& Sonsoles, 2006, citado en Patrón-Espinosa, 2013).

La actual clasificación de heridas afectivas que surgen en otros ámbitos es la siguiente:

La muerte de un ser querido: debido a que la muerte de un ser querido significa la ruptura del vínculo con una persona amada, esta herida influye en las creencias y pensamientos que tiene la persona sobre la vivencia del dolor interior; por ejemplo: negando la ocurrencia del hecho, pensando en lo que pudo haber hecho diferente para evitar la pérdida, sintiéndose culpable (Pérez \& Atehortua, 2016). Las personas que no enfrentan ni reconcilian esta herida adecuadamente pueden presentar dificultades físicas, psicológicas y espirituales, como síntomas depresivos, ansiedad, insomnio, dolor agudo, estreñimiento, infartos, entre otros (Rodríguez-Âlvaro, 2019).

Abuso sexual o violación: es una herida que afecta el bienestar físico, psíquico, social y espiritual de la persona, produciendo daños profundos y duraderos, ya que es una experiencia difícil de integrary asimilar por la persona. En relación con el área psicológica, las personas que sufren este tipo de heridas pueden presentar diversas secuelas, que pueden surgir a corto o a largo plazo. De acuerdo a Girón (2015), a corto plazo, las niñas suelen manifestar síntomas ansioso-depresivos, 
y los niños fracaso escolar y dificultades de socialización. A largo plazo, un abuso de este tipo genera en los niños una sensación de impotencia y falta de control que los puede llevar a conductas dañinas en el futuro, como serían los trastornos alimenticios, la delincuencia y el abuso de sustancias (Hornor, 2010). Adicionalmente, esas personas crecen teniendo una incorrecta valoración de ellas mismas y conductas sexualizadas.

Experiencias sexuales tempranas: existen algunas experiencias sexuales tempranas no asimiladas que podrían ser fuente de heridas afectivas, especialmente cuando ocurren en una etapa en la que no existe madurez suficiente (Del Castillo, 2016). Algunas de dichas experiencias son: la pornografía, el autoerotismo, ver o escuchar a los padres teniendo relaciones íntimas, relaciones sexuales en temprana edad y el acoso sexual. Por ejemplo, Velasco y Gil (2017) describen que algunas de las consecuencias que puede generar la exposición a la pornografía son la adicción y dependencia a la misma, depresión, sentimientos de culpa, ira y vergüenza, aislamiento social, dificultades en las relaciones de pareja, percepciones distorsionadas con respecto a la sexualidad y al concepto de hombre y mujer. Así mismo, el consumo de pornografía está relacionado con la masturbación, la cual a su vez podrá originar síntomas ansiosos, depresivos, dificultades en la atención, dificultades en el sueño, problemas digestivos, entre otros (Velasco \& Gil, 2017).

Fracaso en amistades o relaciones interpersonales: herida que surge desde el dolory trauma que ha causado el fracaso en alguna amistad o relación interpersonal significativa (Del Castillo, 2016). La persona que presenta esta herida, cree que posee alguna característica especial que lo hace sentirse diferente, y por ello, las demás personas expresan desinterés hacia ella; generando sentimientos de soledad e inseguridad, y una sensación de no identificación con ningún grupo, especialmente cuando ha sufrido el rechazo por sus pares en la etapa infantil (Young et al., 2012). De ahí que, esta herida puede obstaculizar el desarrollo de habilidades sociales por miedo al rechazo, dificultades a la hora de afrontar conflictos, expresar sentimientos y pensamientos, y conductas de aislamiento, manifestando disconformidad en el trato con los demás; siendo un impedimento para el desarrollo adecuado de su identidad y del crecimiento personal (Calero et. al., 2018).

Ruptura amorosa: herida que surge como consecuencia del dolor y sufrimiento que genera el rompimiento definitivo con el enamorado o enamorada. De acuerdo con Izurieta Sánchez (2015), las consecuencias psíquicas y físicas de una ruptura amorosa pueden ser similares a aquellas que se viven cuando se atraviesa un duelo por muerte: llanto, labilidad emocional, alteraciones en la alimentación y alteraciones del sueño. Por lo tanto, la ruptura amorosa lleva a que la persona se enfrente a las fases del duelo descritas por Kübler-Ross (1969). Las personas que no logran asimilar la ruptura y elaborar el duelo correctamente pueden quedar con una pobre valoración de sí mismo, con baja autoestima y con 
miedo de tener formar otras relaciones (Del Castillo, 2016).

\section{Experiencias dolorosas en la relación} de pareja: esta herida se produce cuando la persona vive experiencias y problemas con su pareja que pueden generar dificultades notables en sus dimensiones física, psicológica y espiritual. Situaciones como la infidelidad, separación o divorcio, violencia, enfermedad grave, infertilidad, adicciones y viudez (Del Castillo, 2016) pueden generar consecuencias en las distintas dimensiones que acaban afectando la relación con uno mismo, con el otro, con el mundo y con Dios.

Bullying (acoso escolar): de acuerdo a Arroyave (2012), el bullying es un comportamiento agresivo que implica un desbalance de poder ejercido en forma intimidatoria, con intención de causar daño al más débil, el cual es repetido en el tiempo y va a aumentando en intensidad. Esta herida, por tanto, aparece cuando la persona ha sido víctima de este maltrato, ya sea por alguno de sus pares o por un grupo de ellos. La herida suele manifestarse a través de diversas conductas de ansiedad, labilidad emocional, humor inestable, ataques de ira, somatizaciones, nerviosismo, pesadillas y conductas de infantilización y dependencia; a largo plazo. Esta herida puede producir consecuencias sociales y emocionales en la edad adulta, aumentando las posibilidades de desarrollar ansiedad y depresión (Lugones \& Ramírez, 2017). También, Lugones y Ramírez (2017) describen que las víctimas de acoso pueden presentar muchas conductas antisociales y delictivas.
Fracaso en el ámbito académico o laboral: herida que surge como consecuencia de un fracaso o dificultad personal en el ámbito académico o laboral (Del Castillo, 2016). El fracaso académico, está asociado no sólo con factores cognitivos, sino con otros factores, en el ámbito personal como la motivación y la falta de esfuerzo; factores familiares como el nivel educativo de los padres y su nivel económico; factores relacionados con el sistema educativo como la preparación de los docentes (Antelm et al., 2018). Las personas que han experimentado situaciones relacionadas con el fracaso académico presentan sentimientos de tristeza, angustia, ira, se perciben con pocas capacidades cognitivas, un autoconcepto negativo en relación con su proceso de aprendizaje, debido a que dicho suceso se percibe como la confirmación de la percepción distorsionada que tiene de sí mismo, disminuyendo aún más su motivación; lo cual puede llevar a deserción escolar, convirtiéndose en un factor de riesgo de exclusión laboral en el futuro (Zamudio et al., 2019).

\section{Limitaciones o fracasos en lo inte-} lectual: hace referencia a la herida que se produce cuando hay una percepción personal de que se es tonto o ignorante, habiendo una incapacidad o dificultad intelectual real, o no (Del Castillo, 2016). Dweck (2015) afirma que la manera en que las personas perciben sus habilidades juega un papel importante en su motivación y en sus logros, y que es posible aumentar la frecuencia de los logros si se tiene una perspectiva de que la inteligencia puede ser desarrollada; en otras 
palabras, si se tiene una "mentalidad de crecimiento". Así mismo, se ha visto que el desarrollo de esta herida depende bastante del antiguo o tradicional concepto de inteligencia, es decir, aquellas personas que no saben que pueden sobresalir en otrasáreas que no son estrictamente académicas, ya que hoy se sabe que existen distintos tipos de inteligencia, pueden desarrollar problemas psicológicos.

\section{Las distintas situaciones que han} llevado a la persona a percibirse o sentirse como un "inútil": hace referencia a heridas que surgen a partir de experiencias en las que la persona se ha etiquetado a sí misma como "inútil" o "incapaz". De acuerdo a Ellis (2010), esto ocurre cuando la persona hace una auto-evaluación condicional y dependiente. En este tipo de evaluaciones se pone una "nota" a lo que se hace, puede haber un otro con el que se compara o compite, o se cometen errores por la misma naturaleza falible del hombre. Este tema pasa por la recta valoración que uno mismo tiene ante cuestiones prácticas y de la vida cotidiana (Del Castillo, 2016), ya que genera una percepción de "incapacidad” y, como resultado, lleva a la persona a definir su valor de acuerdo a su capacidad para ser útil. Cuando se construye una valoración personal condicionada en las "capacidades” personales o en estándares mágicos e irreales, se caerá frecuentemente en problemas emocionales (Ellis, 2010).

Rechazo a su cuerpo: herida que surge cuando la persona se percibe menos valiosa que los demás porque le disgusta determinada parte de su cuerpo, o porque hay personas que la han juzgado o maltratado por dichas características. Cuando una persona no acepta esa parte de su cuerpo, percibe que en su aspecto físico hay algo que no está bien, generando sentimientos de inferioridad, llegando a creer que no merece ser aceptado por los demás, impidiendo el desarrollo de una recta valoración personal (Del Castillo, 2019). De esta manera, en el camino por cumplir con ciertos estereotipos sociales, se pueden generar perturbaciones en relación con la percepción del propio cuerpo, y puede llevar al desarrollo de trastornos relacionados con la imagen corporal, como el Trastorno Dismórfico Corporal o los Trastornos de la Conducta Alimentaria (Behar \& Arancibia, 2015). A su vez, esto puede generar síntomas ansioso-depresivos, especialmente en aquellas personas que se sienten gravemente perjudicadas por su corporalidad, y se culpabilizan por sentir rechazo hacia sí mismos.

\section{Rechazo a su raza o realidad socioe-} conómica: según Smith et al. (2010) hace referencia a las heridas que aparecen en la vida de las personas que alguna vez han sido excluidas o vulnerabilizadas por su raza, estrato social o realidad económica. Son personas que se han visto despreciadas por los demás debido a su realidad cultural y socioeconómica, en otras palabras, que han sido víctimas de la dimensión conductual del prejuicio. Como consecuencia, las experiencias de estigmatización generan en la persona sentimientos de enojo, humillación, insatisfacción personal, tristeza y una valoración negativa de sí mismo (Smith et al., 2010). 
Fracaso en la vida de fe o religión: herida que aparece cuando un creyente tiene fuertes dudas con respecto a la fe, hasta llegar a la angustia; las dudas pueden ser respecto a la existencia del ser supremo, de la vida eterna, etc. Este tipo de heridas puede generar un profundo malestar, especialmente en la dimensión espiritual de la persona, y podrán surgir a partir de decepciones profundas de personas religiosas, experiencias traumatizantes en ceremonias paganas o experiencias intensas negativas con su iglesia, religión o líderes de las mismas (Del Castillo, 2016).

\section{Decepción o infidelidad en la vida} consagrada o religiosa: según Del Castillo (2016) se refiere a las situaciones en las que el religioso/a no le va bien en la comunidad o con su superior, cuando el sacerdote diocesano no se entiende con el obispo o cuando tiene un fracaso en su ministerio apostólico como párroco o como animador vocacional o por acontecimientos relacionados al abuso de autoridad, maltrato físico o psicológico, abuso sexual, entre otros. También, estas heridas pueden surgir a raíz de una situación de infidelidad a los votos de consagrado como el de celibato y obediencia (Del Castillo, 2016). Es una herida que suele tener consecuencias especialmente serias en la dimensión psicológica y espiritual de la persona.

Aborto: heridas o secuelas psicológicas que surgen en la mujer tras haber tenido un aborto inducido, ya que se convierte en un evento estresante en su vida e incluso puede percibirse como una experiencia traumática, además de que puede incluir respuestas relacionadas al proceso de duelo por la pérdida de una vida humana; todo lo cual se denomina "síndrome postaborto" (Fernández, 2015). Algunas de las manifestaciones que se presentan son: sentimientos de culpa, vergüenza, tristeza, angustia, agresividad e irritabilidad, dificultades de adaptación ante el estrés, poca valoración personal, dificultades en la relación con los miembros de la familia, e incluso ideación suicida; los cuales suelen reaparecer un año después del suceso o ante eventos críticos; desencadenando en algunos casos trastornos del estado de ánimo y ansiedad, trastornos alimenticios, trastornos del sueño, entre otros (Prada et al., 2015). La intensidad y el tipo de consecuencias dependerán a su vez, de factores como las características de personalidad, estilos de afrontamiento, la naturaleza del aborto, el vínculo afectivo con la pareja, el estado psicológico previo al suceso, etc. (Fernández, 2015).

\section{Discusión}

Las heridas afectivas se presentan como una dificultad para la persona debido a que suelen generar ideas distorsionadas, esquemas disfuncionales y otro tipo de problemáticas (Del Castillo, 2016) que no permiten que ésta viva de acuerdo a su potencial. Estas ideas o esquemas disfuncionales van orientando a la persona a ver el mundo de una determinada manera, muchas veces retroalimentando un patrón sesgadoy poco objetivo de entender la realidady deafrontarla adecuadamente (Young, 2012). Al identificar el tipo de herida afectiva que pudo haberse generado a lo largo de la 
historia personal, terapeuta y consultante pueden tener una mejor comprensión de los pensamientos, emociones y comportamientos que predominan en la actualidad. Clasificar las heridas y ponerle nombre a la que trae el consultante a sesión, sirve para entender dequé forma sus patrones disfuncionales cumplen con la función de hacer frentea esa herida, ya que no secuentan con los recursos para manejarlay reconciliarla de forma sana por sí mismo.

El objetivo principal del presente artículo era demostrar a partir de la revisión de literatura científica el estado actual de conocimiento acerca de las heridas afectivas, y las consecuencias psicológicas significativas que generanen los distintosámbitos delapersona adulta. A partir de lo que se ha presentado en este trabajo, se puedeargumentar que las heridas afectivas son situaciones que tienen el potencial de producir un impacto en la psique, ya que el dolor que estas producen puede llegara desencadenar pensamientos, emociones y conductas disfuncionales que desarrollan y mantienen el malestar de la persona (Shapiro, 2013). Además, a partirde los estudios revisados, se ha hecho posible clasificar las heridas en dos grandesámbitos según el área en la cual se producen, y según las similitudes encontradas en las consecuencias o manifestaciones de cada herida.

En cuanto a las heridas afectivas familiares, podemos concluir que, al día de hoy, es posible encontrar una diversidad de literatura acerca de los efectos que estas producen en las personas. Del Castillo (2016) argumenta que las heridas familiares afectan, especialmente, el desarrollo del autoconcepto y la autoestima de los niños, ya que se producen cuando hay una necesidad afectiva que no ha sido satisfecha adecuadamente. Deforma similar, Godbout et al. (2019), explican que estas situaciones generan en el niño una creenciadequeson imperfectos, inaceptables y no-merecedores del amor de los demás. A la larga, estas creencias van desarrollando tendencias deansiedad porabandonooevitación de la intimidad (Godbout et al., 2019) y síntomas depresivos y ansiosos (Nuñez et al., 2017). Finalmente, Baader (2014) añade también que, cuando las problemáticasen la familia son especialmentegraves, las heridas desencadenan distintas consecuencias psicológicas a largo plazo, como desregulación de los afectos, dificultades para identificar y expresar las emociones, y una incapacidad para desarrollar conductas de empatía.

Con respecto a las heridas afectivas de otros ámbitos, es posible concluir que también tienen la potencialidad degenerar dificultades en las personas, y que este tipo de malestarmuchasveces sevivede forma másintensa y duradera (Luciano \& Sonsoles, 2006, citados en Patrón-Espinosa, 2013). En el caso de la muerte de un ser querido, por ejemplo, se ve como un duelo que no ha sido resuelto puede desarrollar tristezay sentimientos de culpa ante la pérdida (Pérez \& Atehortua, 2016), síntomas similares a los de las heridas originadas poruna rupturaamorosa (Izurieta Sánchez, 2015). Por otro lado, las diversas experiencias sexuales que producen heridas afectivas suelen manifestarse por medio de síntomas ansiosos, depresivos, dificultades en la atención y en el sueño (Velasco \& Gil, 2017). Honor (2010) añadeque, a largo plazo, estas heridas son el origen de una sensación de impotencia y falta de control que los puede llevar a conductas perjudiciales, 
comolos trastornos deconductaalimentaria, las conductas delictivas y la dependencia a sustancias. Las heridas interpersonales, comoel fracaso de una amistad o el acoso en el área académica o laboral, son el origen de autoconceptos negativos en las personas, y además desarrollan sentimientos de soledad einseguridad, una sensación estigmatización (Young et al., 2012) y consecuencias sociales y emocionales en la edad adulta, las cuales aumentan la probabilidad de desarrollar ansiedad y depresión (Lugones \& Ramírez, 2017). Aquellas heridas referentes a la intelecto y a la "capacidad personal", que según Ellis (2010) y Dweck (2015) se desarrollan a partir de las evaluaciones personales y el concepto tradicional de inteligencia, suelen tener un impacto directo en el desarrollo adecuado del autoconcepto y la valoración personal (Del Castillo, 2016). Las heridas afectivas que surgen de distintos tipos de rechazo, comoal cuerpo, a la raza o a la realidad socioeconómica, suelen causar enojo, humillación, insatisfacción personal, tristeza y una pobre valoración personal (Smith et al., 2010), que si se prolongan, pueden llegar a generarcuadros clínicosgraves. Las heridas quesurgen en lavida defe, tanto para religiosos como para laicos, suelen afectar tanto la dimensión psicológica como la espiritual (Del Castillo, 2016), teniendo más manifestaciones en la última mencionada. Finalmente, se puedeconcluirtambiénquela herida afectiva que puede aparecer desde un aborto tiene consecuencias que se acercan a la vertiente del trastorno depresivo (Fernández, 2015) y que, por tanto, si no se manejan, pueden manifestarse también a muy largo plazo.

En definitiva, se puede argumentar que los méritos de este trabajo son significativos porque dan respuesta y facilitan el trabajo llevado a cabo en el primer paso del proceso de reconciliación personal, el mismo que hacereferenciaa la identificación dela herida afectiva (Del Castillo, 2016). Al proponer una clasificación más ordenaday concreta de los tipos de heridas afectivas, se hace más fácil que terapeuta y consultante puedan identificarlas correctamentey que, además, sepan las problemáticas de la vida del consultante que podrían tener queabordara raíz de dicha herida. De acuerdo a la Psicoterapia de la Reconciliación, cuando se han trabajado estas dificultades la persona tiene una base más fuerte para continuar con el proceso de reconciliación personal, en el quetendráque enfrentarse a la aceptación, el perdón y la reconciliación con el ofensor. Este proceso, sustentado en la correcta identificación de la herida afectiva, ayuda a que los cambios terapéuticos logrados con las técnicas de otras corrientes se vuelvan más duraderos y estables en el tiempo.

\section{Limitaciones y futuras líneas de investigación}

En cuanto a las limitaciones de esteartículo, sepuedeconsiderar, en primer lugar, queaún hay muy pocas publicaciones que estudian el impacto de las heridas afectivas, ya que es un término psicológico que se ha desarrollado hace menos de 10 años. En concreto, la primera clasificación de heridas afectivas quese publicó fueen el libro "Reconciliación de la historia personal” (Del Castillo, 2016).

Una segunda limitación sería que en la presente revisión solo se tomó en cuenta las consecuencias de las heridas afectivas en la población adulta. Se ha visto 
que el modelo de la Psicoterapia de la Reconciliación puedeseraplicado desdelos 7 años en adelante, ya que muchos niños y adolescentes también se pueden enfrentar a algunas de las heridas mencionadas y pueden tener dificultad para manejarlas adecuadamente. Sin embargo, la mayoría de casos que han sido abordados con este enfoque han sido de adultos, y por tanto se desconoce las consecuencias que estas heridas pueden tener en la población infantojuvenil y las herramientas que serían óptimas para abordarlas.

A partir de lo que se ha comentado en el apartadoanterior, y basado en las limitaciones que se han encontrado, se recomiendan las siguientes futuras líneas de investigación sobre este tema. En primer lugar y a partir de los resultados encontrados, se considera necesario no sólo establecer una clasificación completa de las heridas afectivas, sino también diseñar alguna herramienta de screening que permita a los profesionales identificar con rapidez el ámbito y el tipo de herida que presenta el consultante. Esto podría orientar la entrevista del consultante y facilitar el reconocimiento del recuerdo o evento no-procesado que le produce dolor en la actualidad.
En segundo lugar, sería interesante ahondar un poco más en los tipos de heridas afectivas, e investigar la recurrencia de cada herida en poblaciones específicas. Por poblaciones específicas podríamos entendergrupos de refugiados, grupos de consagrados, grupos que vivieron el conflicto armado en Colombia y en Venezuela, familias con miembros diagnosticados con un trastorno mental grave, familias con miembros diagnosticados con enfermedades crónicas, entre otros.

Por último, y según la demanda que puede surgir en el ámbito clínico, se recomienda estudiara profundidad las heridas quetienen una alta probabilidad de afectar a niños y adolescentes, para así poder elaborar una clasificación apropiadaa su edady desarrollar herramientas y técnicas que pueden ser de uso para abordarel proceso de reconciliación personal con ellos.

\section{Financiamiento}

El presente trabajo fue autofinanciado.

\section{Conflicto de intereses}

Los autores declaran no tener conflicto de interés. 


\section{Referencias}

Antelm, A., Gil, A. Cachaeiro, M., \& Pérez, E. (2018). Causas del fracaso escolar: un análisis desde la perspectiva del profesorado y el alumnado. Enseñanza $\mathcal{E}$ Teaching, 36(1), 129-149. https://revistas.usal.es/index.php/o212-5374/article/ view/et2018361129149/19128

Arroyave, P. (2012). Factores de vulnerabilidad y riesgo asociados al bullying. Revista CES Psicología, 5(1), 118-125.

Baader, B. (2014). Niños y niñas expuestos/as a violencia intrafamiliar. http://repositorio.uchile. cl/bitstream/handle/2250/134567/Christel\%2oBaader.pdf

Behar, R., \& Arancibia, M. (2015). Trastornos de la imagen corporal: anorexia nerviosa versus anorexia inversa (trastorno dismórfico muscular). Revista Mexicana de Trastornos Alimentarios, 6(2), 121-128. http://www.scielo.org.mx/scielo. php?pid=S2007-15232015000200121\&script=sci_arttext

Bourbeau, L. (2014). Las 5 heridas que impiden ser uno mismo. 2da ed. Ob Stare.

Bradburn, N. M. (1969). The structure of psychological well-being. Aldine.

Calero, A., Barreyro, J., Formoso, J., \& Injoque-Ricle, I. (2018). Inteligencia emocional y necesidad de pertenencia al grupo de pares durante la adolescencia. Revista Subjetividad y Procesos Cognitivos, 22(2). http://dspace.uces.edu.ar:818o/xmlui/ bitstream/handle/123456789/4683/Inteligencia_Calero_otros.pdf?sequence=1

Camps, D. (2007). El artículo científico: Desde los inicios de la escritura al IMRYD. Archivos de Medicina, 3(5). https://www.archivosdemedicina.com/medicina-de-familia/el-artculo-cientfico-desde-los-inicios-de-la-escritura-al-imryd.pdf

Del Castillo, H. (2016). Reconciliación de la historia personal. 2da Edición. Areté: Centro de Desarrollo Integral de la Persona.

Del Castillo, H. (2019). Creciendo en la valoración personal día a día. Areté: Centro de Desarrollo Integral de la Persona.

Dweck, C. (2015). Carol Dweck Revisits the "Growth Mindset". Education Week, 35(5), 20-24. 
Ellis, A. (2010). Overcoming destructive beliefs, feelings, and behaviors: New directions for rational emotive behavior therapy. Prometheus Books.

Fernández, E. (2015). El aborto terapéutico o cualquier tipo de aborto, ¿una cuestión de decisión personal en búsqueda de un bien? Secuelas psicológicas. UCV-HACER, 4(2), 148-156. http://revistas.ucv.edu.pe/index.php/UCV-HACER/ article/view/744/583

Figueroa, R. A., Cortés, P. F., Accatino, L., \& Sorensen, R. (2016). Trauma psicológico en la atención primaria: orientaciones de manejo. Revista Médica de Chile, 144(5), 643-655.

Forero, L. C. A., Araújo Reyes, A. P., Godoy Díaz, A. P., \& Vera Rueda, M. E. (2010). Maltrato infantil y sus consecuencias a largo plazo. MedUNAB, 13(2), 103-115. https://revistas.unab.edu.co/index.php/medunab/article/view/1155

García del Junco, J., \& Castellanos Verdugo, M. (2007). La difusión de las investigaciones y el formato IMRYD: Una pesquisa a propósito de la lectura crítica de los artículos científicos. Acimed, 15(1).

Girón, R. (2015). Abuso sexual en menores de edad, problema de salud pública. Avances en Psicología, 23(1), 61-71.

Godbout, N., Daspe, M. Ė., Runtz, M., Cyr, G., \& Briere, J. (2019). Childhood maltreatment, attachment, and borderline personality-related symptoms: Gender-specific structural equation models. Psychological Trauma: Theory, Research, Practice, and Policy, 11(1), 90.

Hornor, G. (2010). Child sexual abuse: Consequences and implications. Journal of Pediatric Health Care, 24(6), 358-364.

Izurieta Sánchez, C. (2015). Proceso de elaboración de duelo en rupturas amorosas de jóvenes de 18 a 25 años. (Tesis de bachillerato) Universidad de las Américas, Quito, Ecuador.

Koss, K. J., George, M. R., Bergman, K. N., Cummings, E. M., Davies, P. T., \& Cicchetti, D. (2011). Understanding children's emotional processes and behavioral strategies in the context of marital conflict. Journal of Experimental Child Psychology, 109(3), 336-352.

Kübler-Ross, E. (1969). On death and dying. The Macmillan Company. 
Kleponis, P. (2018). Pornografía. Comprender y afrontar el problema. Voz de Papel.

Lugones, M., \& Ramírez, M. (2017). Bullying: aspectos históricos, culturales y sus consecuencias para la salud. Revista Cubana de Medicina General Integral, 22(1), 154-162.

Ministerio de Sanidad, Servicios Sociales e Igualdad. (2018). Clasificación Internacional de Enfermedades. n1ava ed. Madrid.

Nuñez, C. S., Pérez, C., \& Castro, M. (2017). Consecuencias del divorcio-separación en niños de edad escolar y actitudes asumidas por los padres. Revista Cubana de Medicina General Integral, 33(3), 296-309.

Packer, A. L., Biojone, M. R., Antonio, I., Takenaka, R. M., Pedroso García, A., Silva, A. C. D., ... \& Delbucio, H. C. R. F. (2001). SciELO: una metodología para la publicación electrónica. Revista Española de Salud Pública, 75(4), 291-312.

Papalia, D. E., \& Martorell, G. (2012). Desarrollo Humano. McGraw-Hill.

Pubmed Central (s/f) US National Library of Medicine National Institutes of Health. NCBI. https://www.ncbi.nlm.nih.gov/pmc/?

Patrón-Espinosa, F. D. J. (2013). La evitación experiencial como dimensión funcional de los trastornos de depresión, ansiedad y psicóticos. Journal of Behavior, Health E Social Issues, 5(1), 85-95.

Pérez, D., \& Atehortua, M. (2016). La Comprensión del duelo desde la psicoterapia humanista. Psyconex, 8(12). https://revistas.udea.edu.co/index.php/Psyconex/ article/view/326982/20784208

Plasencia, C. (2008). EBSCO: un recurso de información de excelencia al servicio de los profesionales cubanos. MEDISAN, $12(4), 1-3$. https://www.redalyc.org/ articulo.oa?id=368445249001

Pereda, N., Forns, M., \& Abad, J. (2013). Prevalencia de acontecimientos potencialmente traumáticos en universitarios españoles. Anales de Psicología, 29(1), 178-186.

Polaino, A. (2010). ¿Hay algún hombre en casa? Editorial Desclée de Brouwer, S.A. 
Prada, D., Rojas, D., Vargas, P., \& Ramírez, J. (2015). El aborto en adolescentes, factores de riesgo y consecuencias: revisión de literatura. Salud Areandina, 4(1), 64-77. https://revia.areandina.edu.co/index.php/Nn/article/view/1319/120o

Rodríguez-Álvaro, M. (2019). Impacto del duelo complicado. Una lectura a través del lenguaje del cuidado. Santa Cruz de La Palma, 13(3). http://scielo.isciii.es/ scielo.php?script=sci_arttext\&pid=S1988-348X20190003000o8\&lng=es\&tlng=pt

Ryff, C. D. (1989). Happiness is everything, or is it? Explorations on the meaning of psychological well-being. Journal of Personality and Social Psychology, 57(6), 1069-1081. https://doi.org/10.1037/0022-3514-57.6.1069

Salgado, A. (2014). Revisión de estudios empíricos sobre el impacto de la religión, religiosidad y espiritualidad como factores protectores. Propósitos y Representaciones, 2(1), 121-159. http://dx.doi.org/10.20511/pyr2014.v2n1.55

Samper, E. (2016). Personalidad ante el trauma en el siglo XXI: fragilidad y antifragilidad ante el trauma. Sanidad Militar, 72(3), 209-215. http://scielo.isciii.es/ scielo.php?script=sci_arttext\&pid=S1887-85712016000300007\&lng=es\&tlng=pt

Sarráis, F. (2016) Psicopatología. EUNSA.

Shapiro, F. (2013). Supera tu pasado. Tomar el control de la vida con el EMDR. Kairós.

Smith, V., Moreno, M., Román, N., Kirschman, D., Acuña, M., \& Víquez, S. (2010). Discriminación social, consecuencias psicológicas y estrategias de afrontamiento en miembros de grupos sociales estigmatizados. Dominación, compromiso y transformación social. Universidad de Costa Rica.

Trautmann, A. (2008). Maltrato entre pares o "bullying": Una visión actual. Revista Chilena de Pediatría, 79(1), 13-20.

Universidad de Navarra (2011). Unika: Nuevo buscador unificado de la Biblioteca de la Universidad de Navarra. https://www.unav.edu/en/web/vida-universitaria/ detalle-noticia-pestana/2011/o9/28/unika:-nuevo-buscador-unificado-de-la-biblioteca-de-la-universidad-de-navarra?articleId=302098

Velasco, A., \& Gil, V. (2017). La adicción a la pornografía: causas y consecuencias. Drugs and Addictive Behavior, 2(1), 122-130. https://www.funlam.edu.co/revistas/ index.php/DAB/article/view/2265/1703 
Young, J., Klosko, J. S., \& Janet, J. (2012). Reinventa tu vida. Cómo superar las actitudes negativas y sentirse bien de nuevo. PaidósIbérica.

Young, J., Klosko, J. S., \& Janet, J. (2013). Terapia de Esquemas. Desclée de Brouwer, S.A.

Zamudio, P., López, F., \& Reyes-Sosa, H. (2019). La representación social del fracaso escolar. La hipótesis del núcleo central. Perfiles Educativos, 41(165). http://www. perfileseducativos.unam.mx/iisue_pe/index.php/perfiles/article/view/59051/52256

Zeanah, C. H., \& Humphreys, K. L. (2018). Child abuse and neglect. Journal of the American Academy of Child E Adolescent Psychiatry, 57(9), 637-644.

Recibido: 14 de noviembre de 2020

Revisado: 20 de marzo de 2021

Aceptado: 8 de abril de 2021 